\title{
The Crystallographic and Electronic Phase Diagrams of Yttria-Stabilized Zirconia Model Electrolytes
}

\author{
T. Götsch ${ }^{\mathrm{a}}$, A. Menzel ${ }^{\mathrm{a}}$, E. Bertel ${ }^{\mathrm{a}}$, M. Stöger-Pollach ${ }^{\mathrm{b}}$, and S. Penner ${ }^{\mathrm{a}}$ \\ a Institute of Physical Chemistry, University of Innsbruck, A-6020 Innsbruck, Austria \\ ${ }^{\mathrm{b}}$ University Service Centre for Transmission Electron Microscopy \\ TU Wien, A-1040 Vienna, Austria
}

\begin{abstract}
Yttria-stabilized zirconia model electrolyte systems with four different compositions are analyzed regarding their crystallographic and electronic structure. By investigating the unit cell height, obtained from electron diffraction patterns, it is shown that a phase transformation between the tetragonal and cubic polymorphs takes place between 8 and 9.3 mol\%. Furthermore, the direct band gaps are shown to exhibit the same behavior as the lattice parameter, featuring a discontinuity at the phase transition. By measuring the emitted Čerenkov radiation, an electronic transition that is smaller than the band gaps is found, suggesting that localized defect states are present within the band gap, which is in agreement with UV photoelectron spectra.
\end{abstract}

\section{Introduction}

Yttria-stabilized zirconia (YSZ) is known for its high ionic conductivity at elevated temperatures (1), making it ideal for employment as an oxygen ion conducting electrolyte for applications such as chemical sensors (2) or solid oxide fuel cells (SOFCs) (3). In the case of the latter, it is also used as a component of the anode, which usually consists of a $\mathrm{Ni} / Y S Z$ cermet (4). There, it is required for two tasks: besides facilitating the transport of $\mathrm{O}^{2-}$ from the electrolyte to the three-phase boundary, where the reaction takes place, it also plays a role in the catalytic process involving the activation of the fuel fed to the SOFC.

In both cases, the relevant properties - be it the ionic/electronic conductivity or the catalytic activity - rely on the existence of oxygen vacancies as well as the electronic structure of the solid solution - and, concomitantly, also on the crystallographic structure. However, the number of published experimental studies regarding the electronic structure of YSZ is sparse (5-7). There are some reports on unsubstituted zirconium dioxide, of which most rely on UV/VIS experiments (8-10). But the determination of the optical band gap by this method leads to a large spread in the results (8). Additionally, DFT calculations of densities of states and band structures have been published for pure $\mathrm{ZrO}_{2}$ (11-14), as well as for YSZ (15).

Moreover, there are discrepancies in the $\mathrm{ZrO}_{2} / \mathrm{Y}_{2} \mathrm{O}_{3}$ phase diagrams reported in literature. Existence and extent of the area of stability of some crystallographic phases are controversial. For instance, there are conflicting reports about the $\mathrm{Y}_{2} \mathrm{O}_{3}$ concentration at which the phase transformation between tetragonal and cubic YSZ occurs. 
Inconsistencies arise also concerning the existence of different ordered phases like the $\delta$ phase, $\mathrm{Zr}_{3} \mathrm{Y}_{4} \mathrm{O}_{12}$ (16), a pyrochlore structure $\left(\mathrm{Zr}_{2} \mathrm{Y}_{2} \mathrm{O}_{7}\right)$ (17) or a compound with the formula $\mathrm{ZrY}_{6} \mathrm{O}_{11}$ (18). Regarding the $\delta$ phase, a consensus seems to be in sight, as all more recent phase diagrams contain it. There is, however, still the question of its nature of decomposition at higher temperatures (i.e. whether it transforms peritectoidically or dystectoidically into the cubic solid solution). A more detailed summary of the available literature is given in (19).

Thus, we opted for a systematic investigation of the crystallographic as well as the electronic phase diagrams of YSZ, i.e. the determination of the crystal structure and the electronic structure as a function of the yttria content in the complex oxide. For this, we prepared thin films as model electrolyte systems, containing $3 \mathrm{~mol} \%, 8 \mathrm{~mol} \%, 20 \mathrm{~mol} \%$ and $40 \mathrm{~mol} \% \mathrm{Y}_{2} \mathrm{O}_{3}$, respectively.

\section{Experimental}

\section{$\underline{\text { Thin Film Preparation }}$}

The thin films were deposited using a home-built direct current ion beam sputter gun (19). This source, operated in an Argon background pressure of $5 \times 10^{-5} \mathrm{mbar}$ at an argon ion energy of $2 \mathrm{keV}$, allows for the preparation of electron-transparent thin films (with thicknesses of approximately $25 \mathrm{~nm}$ ) at controlled growth rates, facilitating the epitaxial growth. As substrates, freshly cleaved $\mathrm{NaCl}(001)$ single crystals were used for the transmission electron microscopy samples, which could subsequently be submerged in water to float off the thin film, resulting in well-ordered, unsupported thin films. The photoelectron spectroscopy specimens were deposited on $\operatorname{Si}(111)$ wafers. As targets, commercially available powders from Sigma Aldrich (with concentrations of $3 \mathrm{~mol} \%$, $8 \mathrm{~mol} \%, 20 \mathrm{~mol} \%$ and $40 \mathrm{~mol} \%$ ) were pressed to pellets using a force of $20 \mathrm{kN}$. Throughout this work, these samples will be called 3YSZ, 8YSZ, 20YSZ and 40YSZ, respectively.

\section{Characterization of the Thin Films}

The crystallographic information was collected using transmission electron microscopy (TEM) with a FEI Tecnai F20 S-TWIN (high-resolution) analytical (scanning) transmission electron microscope, operated at $200 \mathrm{kV}$. The microscope was equipped with a GIF Tridiem energy-loss spectrometer and a Gatan Vulcan cathodoluminescence spectrometer that was used for the Čerenkov emission spectroscopy experiments. For the valence electron energy loss spectra (VEELS), the acceleration voltage was lowered to $60 \mathrm{kV}$ in order to avoid Čerenkov losses from masking the onset of the interband transitions.

For UV photoelectron spectroscopy (UPS), a Thermo Scientific MultiLab 2000 spectrometer was employed (with a base pressure in the low $10^{-10} \mathrm{mbar}$ range). The instrument was equipped with a He plasma UV source and an Alpha 110 hemispherical sector analyzer. 


\section{Results and Discussion}

Using XRD, the crystal structures of the targets could be determined (19). According to this, $3 \mathrm{YSZ}$ is a mixture between tetragonal YSZ and monoclinic $\mathrm{ZrO}_{2}, 8 \mathrm{YSZ}$ is purely tetragonal, 20YSZ crystallizes in the cubic polymorph and the sample with $40 \mathrm{~mol} \%$ $\mathrm{Y}_{2} \mathrm{O}_{3}$ is found to be rhombohedral (i.e. the $\delta$-phase, $\mathrm{Zr}_{3} \mathrm{Y}_{4} \mathrm{O}_{12}$ ).
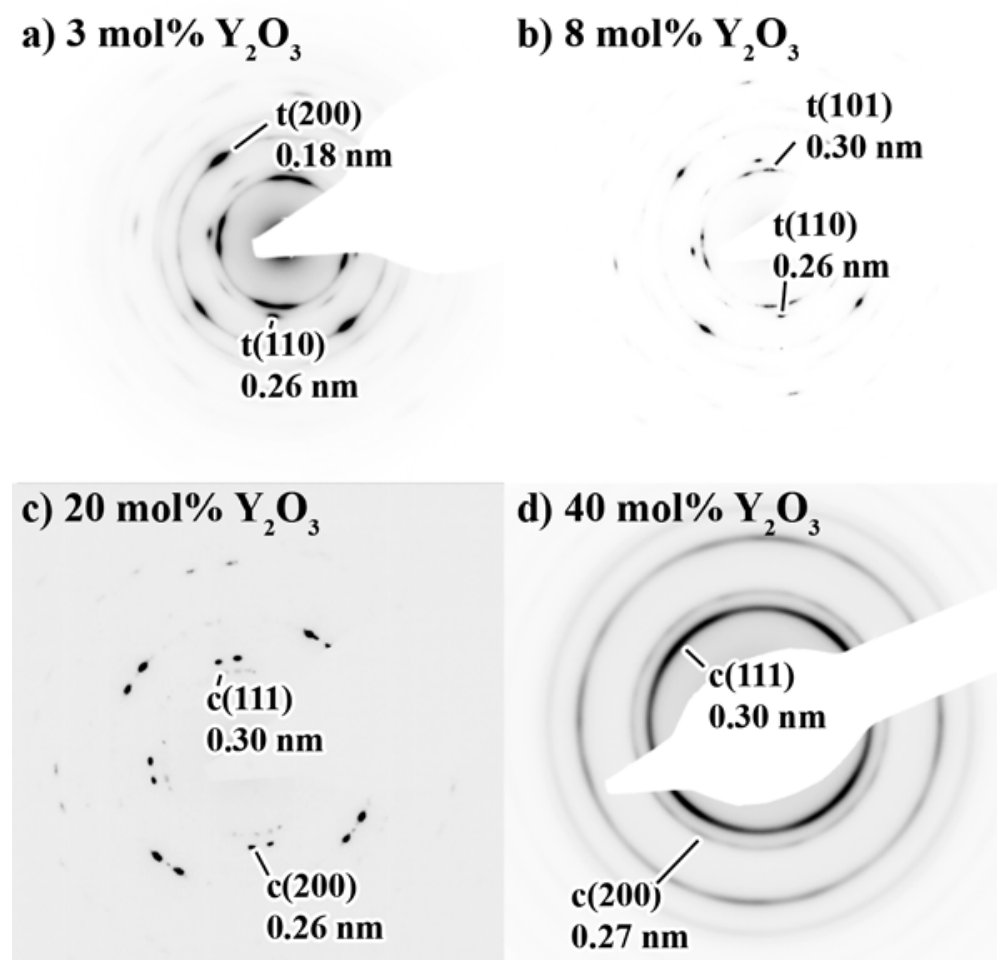

Figure 1. Selected area electron diffraction patterns of a) $3 \mathrm{YSZ}$, b) $8 \mathrm{YSZ}$, c) $20 \mathrm{YSZ}$ and d) 40YSZ. Adapted from (19).

In order to investigate whether a phase transition occurred during the sputtering process, selected area electron diffraction (SAED) patterns were recorded. These are shown in Figure 1. Taking a closer look at the patterns, it can be observed that they all look very similar, containing the same spots and rings with no discernible differences in intensities. This is a problem with zirconium dioxide in general, as the tetragonal and cubic polymorphs exhibit the same diffraction patterns due to the existence of lattice planes with the same spacings (20). Thus, distinguishing them by means of diffraction methods (this also applies to XRD) is difficult. However, the distinction can still be made by exploiting that the heights of the tetragonal and the cubic unit cells are equivalent. This is also seen by the fact that the (002) spots in the diffraction patterns are the same for tetragonal and cubic patterns. Hence, the lattice parameter $c$ can be calculated from these rings, describing the unit cell size (i.e. the height). Furthermore, 4 the same parameter can also be calculated from the other rings, which improves accuracy and reliability (19). During this calculation, it does not matter whether the crystal structure is assumed to be tetragonal or cubic - the results will be the same. However, the isotropy of the cubic cell makes the calculation significantly easier as the $c / a$ ratio is kept fixed at 1. The unit cell height, $c$, can then be calculated via equation 1, with $d_{h k l}$ being the lattice spacing for each plane. 


$$
c=\left[d_{h k l}{ }^{2} \times\left(h^{2}+k^{2}+l^{2}\right)\right]^{1 / 2}
$$

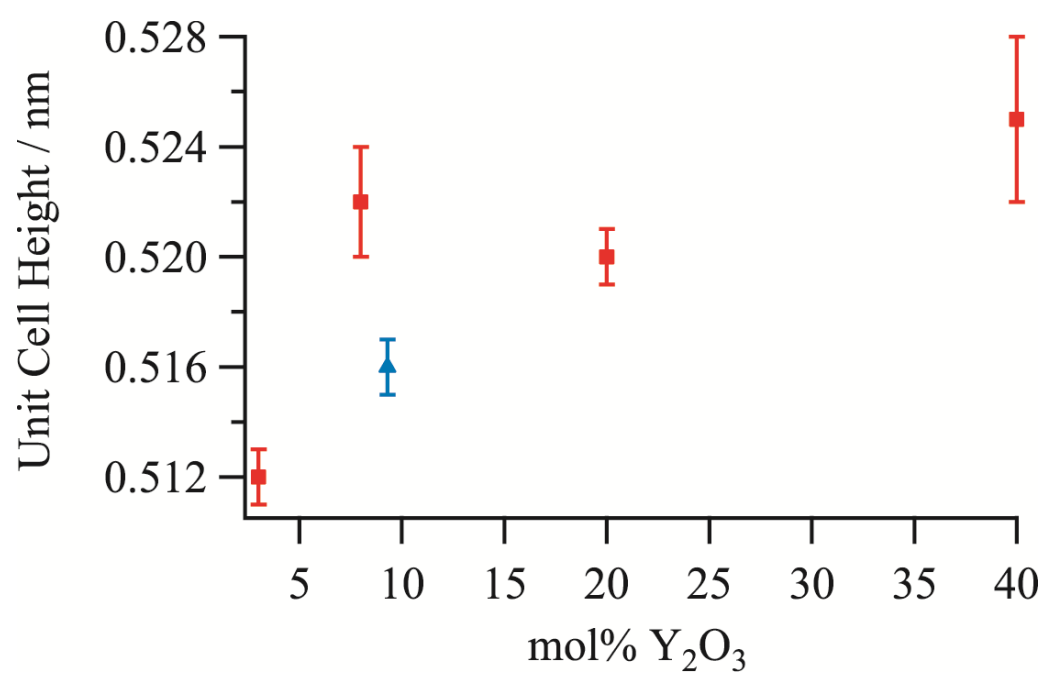

Figure 2. The unit cell height, $c$, as a function of the yttria content reveals the phase transition from tetragonal to cubic to occur between 8 and $9.3 \mathrm{~mol} \% \mathrm{Y}_{2} \mathrm{O}_{3}$. The squares are based on data from (19) and the triangle depicts data from (21).

The resulting unit cell heights for each yttria concentration are displayed in Figure 2. It can be seen that the lattice parameter increases when raising the $\mathrm{Y}_{2} \mathrm{O}_{3}$ content in the solid solution from 3 to $8 \mathrm{~mol} \%$. This is expected since $\mathrm{Y}^{3+}$ is a larger ion than $\mathrm{Zr}^{4+}$; thus, the unit cell volume must increase due to the substitution.

However, going from $8 \mathrm{YSZ}$ to $20 \mathrm{YSZ}$, the lattice parameter shows a decrease. Because of the aforementioned requirement of an increasing cell volume due to the larger yttria concentration, such a behavior signals a lateral lattice expansion (i.e. along $a$ and $b$ ). This means that a phase transformation from the tetragonal to the cubic polymorph takes place between 8 and $20 \mathrm{~mol} \% \mathrm{Y}_{2} \mathrm{O}_{3}$. In fact, in a later experiment using magnetronsputtered thin films, the critical concentration could be pinned down to lie between $8 \mathrm{~mol} \%$ and $9.3 \mathrm{~mol} \% \mathrm{Y}_{2} \mathrm{O}_{3}(21)$. Upon increasing the concentration of the substituting oxide to $40 \mathrm{~mol} \%$, the unit cell expands again.

This shows that the thin films of $8 \mathrm{YSZ}$ as well as 20YSZ show the same crystal structures as the respective sputter targets while $3 \mathrm{YSZ}$ and $40 \mathrm{YSZ}$ are deposited in a different structure. For 3YSZ, it can be understood as a substrate effect: on the (001) surface of the cubic $\mathrm{NaCl}$ single crystal, the tetragonal $\mathrm{YSZ}$ will be preferred as compared to the monoclinic polymorph due to the lower lattice mismatch with the substrate. This can also be seen in the high degree of ordering in the diffraction pattern. This epitaxial growth can also be observed for 8 and $20 \mathrm{~mol} \% \mathrm{Y}_{2} \mathrm{O}_{3}$. The diffraction pattern of 40YSZ, however, does not exhibit such strong texturing. In fact, it looks very polycrystalline, with the pattern being composed of Debye-Scherrer-like rings. This indicates that the deposited cubic polymorph was not induced by the substrate, as that is expected to lead to a higher degree of ordering in the system. It has, however, been demonstrated that the incorporation of argon ions, which inevitably happens during sputtering, stabilizes the cubic polymorph (22). 
a)
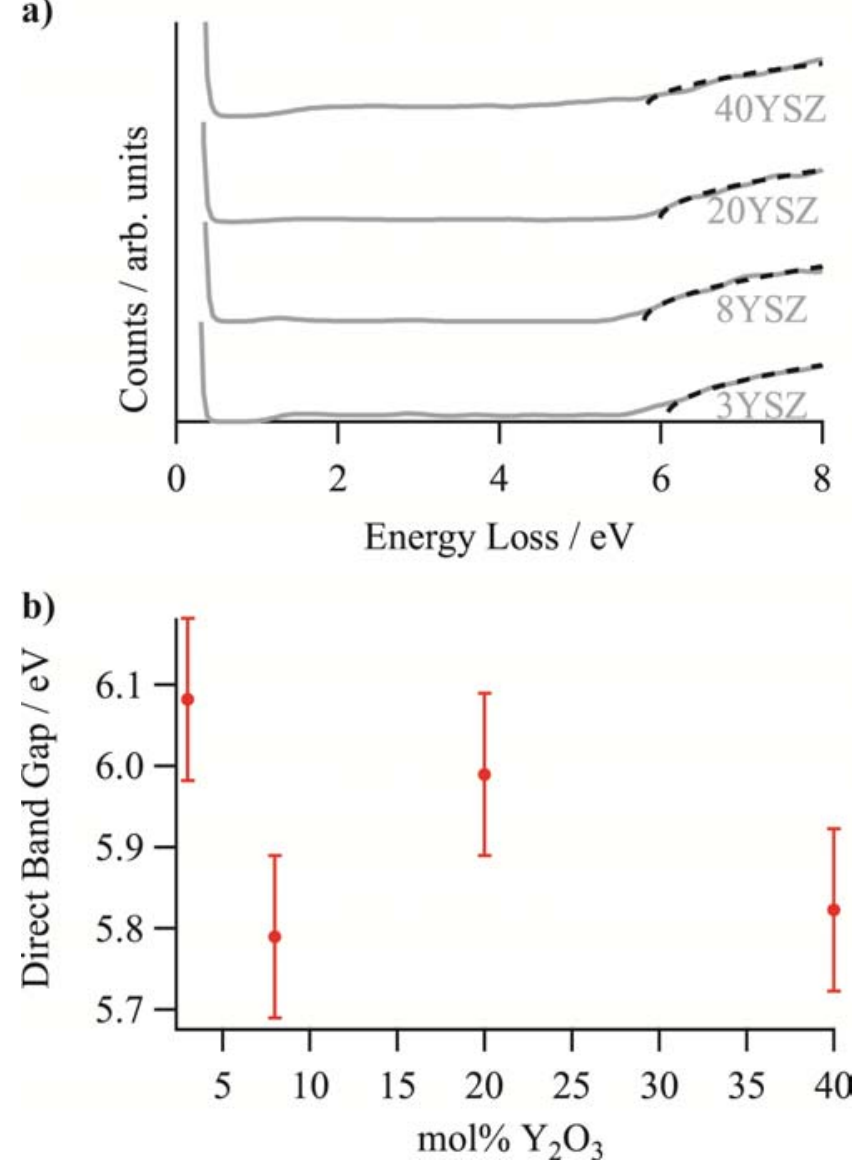

Figure 3. a) VEEL spectra obtained at an acceleration voltage of $60 \mathrm{kV}$. b) Direct band gaps determined from the spectra in a).

Using low-loss EELS, the band gaps can be measured from the onset of the interband transitions. Due to the large difference between the primary energy of the beam and the energy-loss, the dipole approximation is valid $(\Delta k \approx 0)$. Thus, only direct transitions are seen in EELS. However, if the microscope is operated at $200 \mathrm{kV}$, the velocity of the electrons is larger than the vacuum speed of light divided by the index of refraction $(c / n)$. Hence, relativistic losses due to the Čerenkov effect would obscure the onset of the interband transitions (23). To avoid such losses, the acceleration voltage was lowered so that the particles become slow enough to not exceed the speed of light in the sample. This technique is called valence EELS (VEELS) (24).

The spectra acquired in this way are displayed in Figure 3a. Plural scattering was removed from these spectra and a Richardson-Lucy deconvolution algorithm was applied to remove the influence of the tails of the zero-loss peak from the area of interest (25). The slight change in intensity below $2 \mathrm{eV}$ seen in some of the spectra originates from an artifact of this deconvolution procedure and does not represent the band gap. The gap energy was determined by fitting a square root function to the onset of the interband transitions. The results of this procedure are shown in Figure $3 \mathrm{~b}$. The similarity to the trend exhibited by the lattice parameter (Figure 2) is obvious: the band gap also exhibits a discontinuity between 8 and $20 \mathrm{~mol} \% \mathrm{Y}_{2} \mathrm{O}_{3}$. The band gap decreases as the yttria concentration is increased and increases upon the phase transition, thus displaying a reciprocal behavior to the unit cell height. For $3 \mathrm{YSZ}$, the band gap is $6.1 \mathrm{eV}$, whereas it is $5.8 \mathrm{eV}$ for $8 \mathrm{YSZ}$. After the phase transformation, it is $6.0 \mathrm{eV}$ again and drops to $5.8 \mathrm{eV}$ at 
$40 \mathrm{~mol} \%$ yttria. Within one crystallographic phase, the decrease of the band gap is mainly due to the increase in the unit cell volume. Minor effects stem from the altered level of substitution.

a)

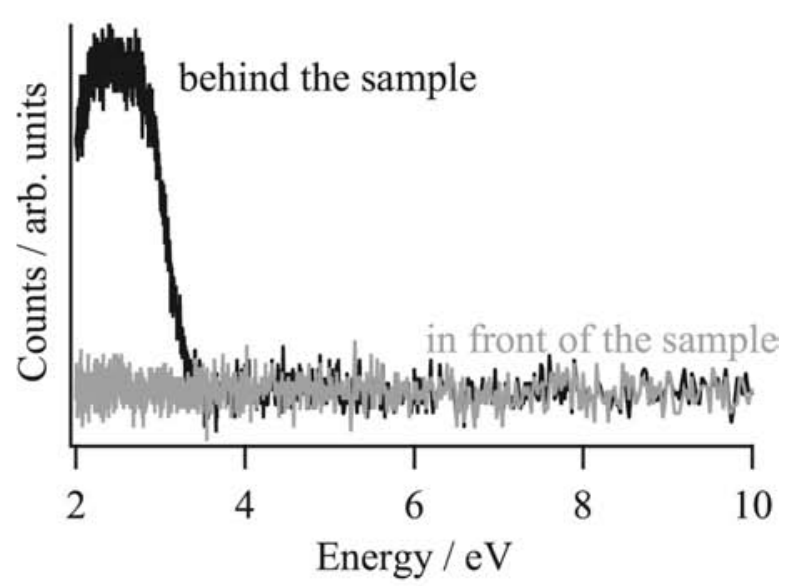

b)

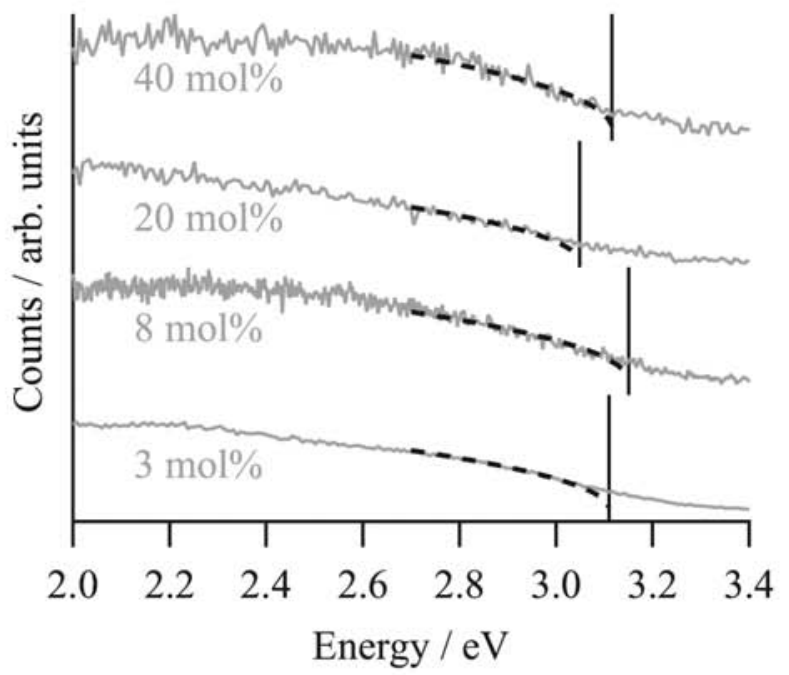

c)

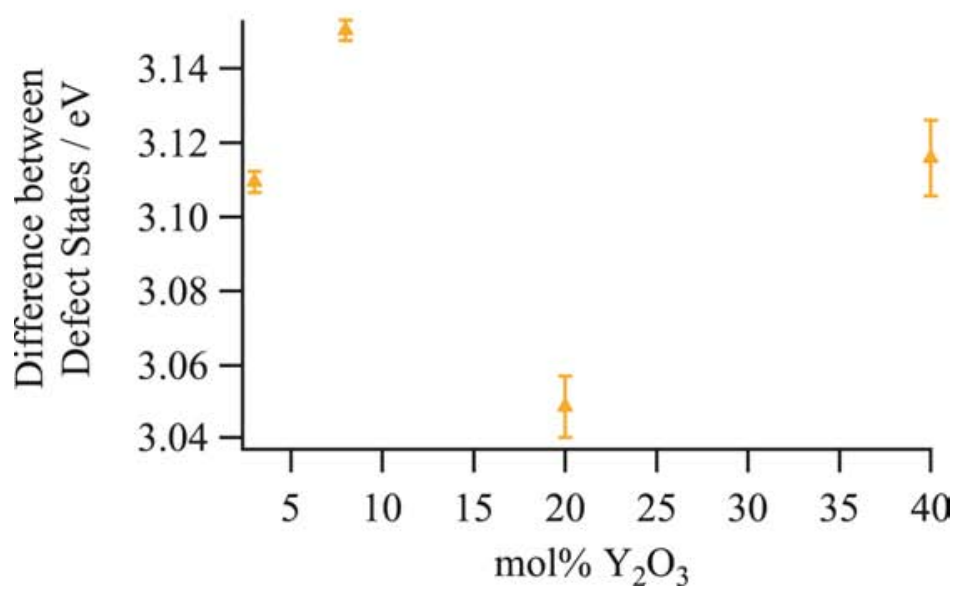

Figure 4. a) The readouts of the two mirrors of the cathodoluminescence spectrometer inside the TEM during the Čerenkov experiments. The Čerenkov emission spectra are given in b), with the concentrations referring to the $\mathrm{Y}_{2} \mathrm{O}_{3}$ content. c) Defect state distances as obtained using Čerenkov emission spectroscopy. 
If, contrary to the VEELS experiments, the acceleration voltage in the TEM is kept at $200 \mathrm{kV}$, thereby deliberately causing the emission of Čerenkov radiation, a cathodoluminescence spectrometer can be used to measure this radiation as it is emitted from the specimen. This is called Čerenkov emission spectroscopy and the spectra can be seen in Figure 4. As stated before, Čerenkov radiation is only emitted in the direction of travel of the charge particle (i.e. the electron). The cathodoluminescence spectrometer used for the detection of the radiation is equipped with two elliptic mirrors (one in front of and one behind the sample), which are read out separately. Comparing the readouts of the two mirrors, one can discriminate Čerenkov radiation from any other source of radiation. This is demonstrated in Figure 4a, showing a signal from the mirror located behind the sample, but only baseline noise for the mirror in front of the sample. The spectra of the four specimens are given in Figure $4 \mathrm{~b}$. All spectra feature an apparent absorption edge at slightly above $3 \mathrm{eV}$, indicating the onset of electronic transitions above $3 \mathrm{eV}$ in all samples. Since the band gap is approximately twice that value, this suggests the presence of defect levels within the band gap that were not observed via VEELS. This is due to the difference in cross-sections between the $60 \mathrm{kV}$ electron beam and the approximately $3 \mathrm{eV}$ photons. The existence of defect states close to the valence band and conduction band edges is in agreement with the findings published by Wiemhöfer et al. (7).

Panel c) of Figure 4 shows the onset energies of the electronic transitions that were measured by Čerenkov emission spectroscopy. This plot again resembles that of the lattice parameter (Figure 2): the energy gap between the defect states increases from $3.109 \mathrm{eV}$ to $3.150 \mathrm{eV}$ upon raising the yttria concentration from 3 to $8 \mathrm{~mol} \%$. For $20 \mathrm{YSZ}$ this value drops significantly to $3.049 \mathrm{eV}$ before increasing again to $3.116 \mathrm{eV}$ at $40 \mathrm{~mol} \%$ $\mathrm{Y}_{2} \mathrm{O}_{3}$. Thus, a higher concentration increases the gap between the defect states, whereas the change in crystal structure decreases it.

In order to independently probe the localized defect states, UV photoelectron spectra were recorded. As UPS samples the valence band, the localized defect states just above the valence band maximum are expected to be detectable. Figure 5 displays the spectra for all samples. All four spectra contain the same features with different intensities. The lowest-energy peak in all spectra stems from the emission of secondary electrons, whereas the emission between 0 and approximately $7 \mathrm{eV}$ originates from the oxygen $2 \mathrm{p}$ states. There is a non-zero density of states above the valence band maximum (VBM). The latter was determined by linear extrapolation of the leading edge of the oxide valence band to the baseline. The density of states above the VBM originates from the localized defects determined by the Čerenkov results. Since the Čerenkov radiation absorption has an onset at 3-3.2 eV, we infer the existence of excited defect states below the conduction band minimum. Accordingly, core-loss EELS or X-ray absorption experiments should exhibit a pre-edge intensity. It will be interesting to test in this way, whether Čerenkov emission spectroscopy as can serve a precise tool for probing defect states in semiconductors and insulators, e.g. the oxides employed in SOFCs. 
a) $3 \mathrm{~mol} \%$

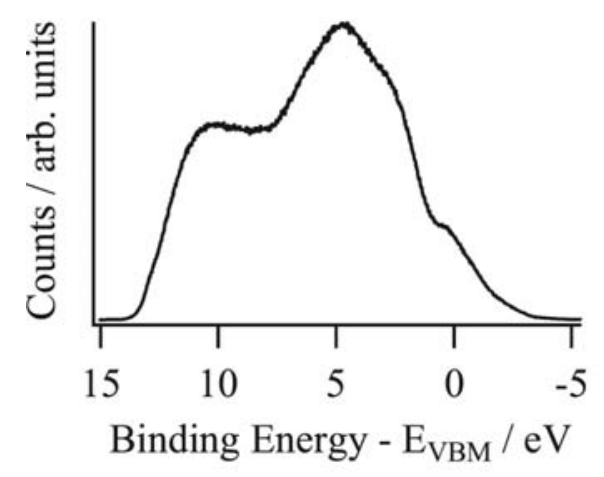

c) $20 \mathrm{~mol} \%$

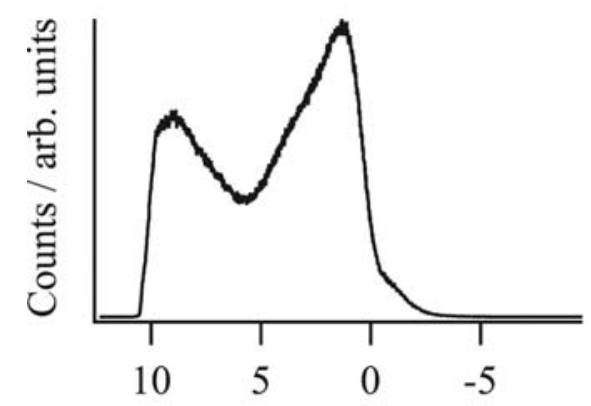

Binding Energy - $\mathrm{E}_{\mathrm{VBM}} / \mathrm{eV}$ b) $8 \mathrm{~mol} \%$

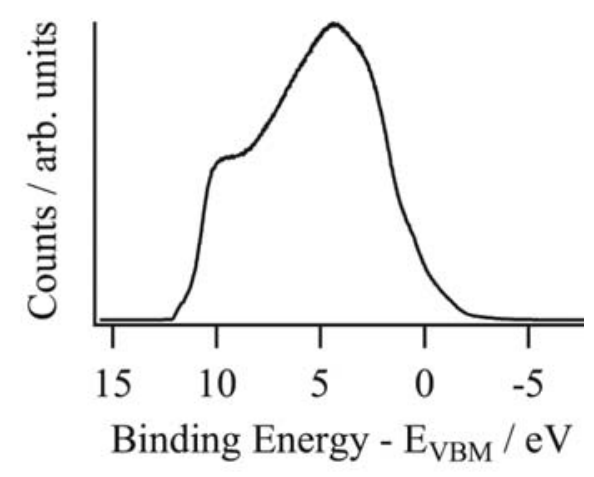

d) $40 \mathrm{~mol} \%$

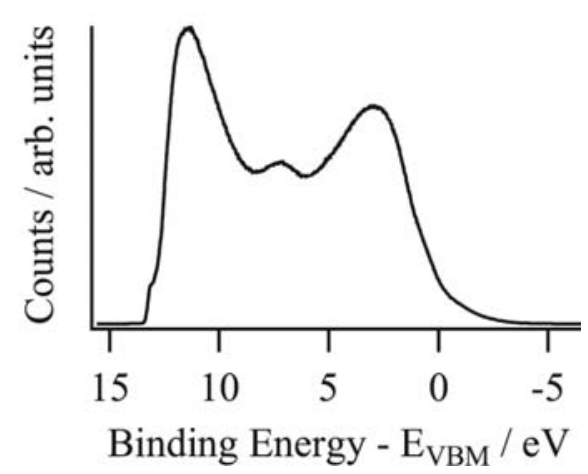

Figure 5. UV photoelectron spectra plotted relative to the valence band maximum (VBM), with the defect states visible.

\section{Conclusion}

A study of the crystallographic phases and the electronic structure of YSZ as a function of the yttria content was presented. By measuring the unit cell heights from electron diffraction patterns, it was shown that the tetragonal-to-cubic phase transition occurs between 8 and 9.3 mol\% $\mathrm{Y}_{2} \mathrm{O}_{3}$. The direct band gaps were calculated from valence EELS data and reflect a reciprocal trend to the unit cell height.

Furthermore, Čerenkov emission spectroscopy revealed the presence of electronic transitions with an onset at about $3 \mathrm{eV}$ - significantly smaller than the band gaps. These transitions most likely stem from excitations at defects and they also feature the same trend as the lattice parameter. UV photoelectron spectroscopy reveals the existence of localized defects just above the valence band maximum and thus corroborates the findings obtained by Čerenkov emission spectroscopy.

The next step required to map out the electronic structure around the band gap is finding the position of the Fermi level within the band gap. For this, X-ray photoelectron spectroscopy is required as the lower photon fluxes reduce the charge-induced shifting of the valence band spectra significantly. Also, using core-loss EELS, the defect states close to the conduction band minimum might be visible. Additionally, the Kramers-Kronig relation can be used to obtain optical properties from the valence EEL spectra, which is currently work in progress, as are theoretical studies using density functional theory. 


\section{Acknowledgments}

This work was funded by the Austrian Science Fund (FWF) via grant numbers F4503-N16 and F4503-N02.

\section{References}

1. T. H. Etsell and S. N. Flengas, Chem. Rev., 70(3), 339-376 (1970).

2. N. Miura, T. Sato, S. A. Anggraini, H. Ikeda and S. Zhuiykov, Ionics, 20(7), 901925 (2014).

3. Steele, Brian C. H. and A. Heinzel, Nature, 414(6861), 345-352 (2001).

4. D. Marinha, L. Dessemond and E. Djurado, Curr. Inorg. Chem., 3(1), 2-22 (2013).

5. N. Nicoloso, A. Löbert and B. Leibold, Sens. Actuators, B, 8(3), 253-256 (1992).

6. G. Garcia, A. Figueras, R. Merino, V. Orera and J. Llibre, Thin Solid Films, 370(1-2), 173-178 (2000).

7. H.-D. Wiemhöfer and U. Vohrer, Ber. Bunsenges. Phys. Chem., 96(11), 16461652 (1992).

8. R. H. French, S. J. Glass, F. S. Ohuchi, Y.-N. Xu and W. Y. Ching, Phys. Rev. B, 49(8), 5133-5142 (1994).

9. C.-K. Kwok, J. Vac. Sci. Technol. A, 8(4), 3345 (1990).

10. E. Fernández López, V. Sánchez Escribano, M. Panizza, M. M. Carnasciali and G. Busca, J. Mater. Chem., 11(7), 1891-1897 (2001).

11. J. C. Garcia, L. M. R. Scolfaro, A. T. Lino, V. N. Freire, G. A. Farias, C. C. Silva, H. W. L. Alves, S. C. P. Rodrigues and E. F. da Silva, J. Appl. Phys., 100(10), 104103 (2006).

12. H. Jiang, R. I. Gomez-Abal, P. Rinke and M. Scheffler, Phys. Rev. B, 81(8) (2010).

13. B. Králik, E. K. Chang and S. G. Louie, Phys. Rev. B, 57(12), 7027-7036 (1998).

14. L. Soriano, M. Abbate, J. Faber, C. Morant and J. Sanz, Solid State Commun., 93(8), 659-665 (1995).

15. S. E. Kul'kova and O. N. Muryzhnikova, Inorg. Mater., 36(1), 38-42 (2000).

16. A. Rouanet, C. R. Seances Acad. Sci., Ser. C, 267(23), 1581-1584 (1968).

17. F.-K. Fan, A. K. Kuznetsov and É. K. Keler, Bull. Acad. Sci. USSR, Div. Chem. Sci. (Engl. Transl.), 12(4), 542-549 (1963).

18. C. Pascual and P. Duran, J. Am. Ceram. Soc., 66(1), 23-27 (1983).

19. T. Götsch, W. Wallisch, M. Stöger-Pollach, B. Klötzer and S. Penner, AIP Adv., 6(2), 25119 (2016).

20. D. G. Lamas and N. E. Walsöe de Reca, J. Mater. Sci., 35(22), 5563-5567 (2000).

21. T. Götsch, T. Schachinger, M. Stöger-Pollach, R. Kaindl and S. Penner, Appl. Surf. Sci., 402, 1-11 (2017).

22. J. Holgado, R. Escobar Galindo, A. van Veen, H. Schut, J. de Hosson and A. González-Elipe, Nucl. Instrum. Methods Phys. Res., Sect. B, 194(3), 333-345 (2002).

23. I. Tamm and I. Frank, Dokl. Akad. Nauk SSSR, 14, 107-112 (1937).

24. M. Stöger-Pollach, Micron, 39(8), 1092-1110 (2008).

25. R. F. Egerton, Electron energy-loss spectroscopy in the electron microscope, New York, Springer (2011). 